\title{
Influence of the supramolecular order on the electrical properties of $1 D$ coordination polymers based materials
}

Chiara Musumeci, ${ }^{\mathrm{a}}$ Silvio Osella, ${ }^{\mathrm{b}}$ Laura Ferlauto, ${ }^{\mathrm{a}, \mathrm{d}}$ Dorota Niedzialek, ${ }^{\mathrm{b}}$ Luca Grisanti, ${ }^{\mathrm{b}}$ Sara Bonacchi, ${ }^{\mathrm{a}}$ Abdelaziz Jouaiti, ${ }^{\mathrm{c}}$ Silvia Milita, ${ }^{\mathrm{d}}$ Artur Ciesielski, ${ }^{\mathrm{a}}$ David Beljonne, ${ }^{\mathrm{b},{ }^{*}}$ Mir Wais Hosseini, ${ }^{\mathrm{c},}{ }^{*}$ Paolo Samori ${ }^{\mathrm{a}, *}$

a ISIS \& icFRC, Université de Strasbourg \& CNRS, 8 allée Gaspard Monge, 67000, Strasbourg, France. E-mail: samori@unistra.fr

${ }^{b}$ Laboratory for Chemistry of Novel Materials, University of Mons, Place du Parc 20, B-7000 Mons, Belgium. E-mail: david.beljonne@umons.ac.be

c Laboratoire de Tectonique Moléculaire, UMR UdS-CNRS 7140, icFRC, Institut Le Bel, Université de Strasbourg, 4 rue Blaise Pascal, CS 90032, 67081 Strasbourg, France. E-mail: hosseini@unistra.fr

${ }^{\mathrm{d}}$ CNR - Istituto per la Microelettronica e Microsistemi (IMM), via Piero Gobetti 101, I-40129 Bologna, Italy. 


\begin{abstract}
The generation, under self-assembly conditions, of coordination polymers on surface based combinations of a terpyridine-antracene-pyridine based tecton and $\mathrm{Co}(\mathrm{II})$ or $\mathrm{Pd}(\mathrm{II})$ cations is primarily governed by the coordination geometry of the metal center (octahedral and square planar respectively). While the octahedral Co(II) based polymer self-assembles in insulating films exhibiting randomly oriented crystalline domains, the planarity of $\operatorname{Pd}(\mathrm{II})$ based polymers leads to the formation of conductive $\pi-\pi$ stacked fibrillar structures exhibiting anisotropically oriented domains. In the latter case, the favorable Pd-Pd and anthracene-anthracene wavefunction overlaps along the fiber direction are responsible for the large electronic couplings between adjacent chains, whereas small electronic couplings are instead found along individual polymer chains. These results provide important guidelines for the design of conductive metal coordination polymers, highlighting the fundamental role of both intra- as well as inter-chain interactions, thus opening up new perspectives towards their application in functional devices.
\end{abstract}




\section{Introduction}

Self-assembly involving suitably designed organic ligands and metal ions represents an effective strategy for the fabrication of novel materials exhibiting sophisticated structural motifs. ${ }^{1}$ During the past years, numerous one- (1D), two- (2D), and three-dimensional (3D) coordination polymers have been generated by combining transition metal templates with mono-, bi- or multidentate organic spacers featuring different rigidity. ${ }^{2}$ The major research focus on such compounds has been oriented towards the generation of porous architectures for gas storage and sensing. ${ }^{3}$ However, the knowledge gathered on the exploration and exploitation of their electrical properties is still relatively poor, ${ }^{4}$ although recently it was demonstrated that metals can efficiently mediate conjugation pathways between organic molecules, leading to new materials with impressive electrical properties, such as conductivity higher than that of organic conductors ${ }^{5}$ and remarkable thermoelectric properties. ${ }^{6}$ By exploiting the modular nature of self-assembly, a judicious choice of the components such as metal ions, peripheral ligands, and modular bridging ligands offers a direct route towards the tailoring of the overall structure in terms of geometry, distance and electronic interaction between the metal centers in a versatile way, enabling the emergence of interesting phenomena such as photoinduced processes, vectorial transport of energy or charges, charge separation and multiredox reactions. ${ }^{7}$ The electrical properties of single stranded coordination polymers have been explored through the fabrication of electrical junction integrating between electrodes either a single molecule (break junction) ${ }^{8}$ or ensembles of molecules grafted on solid conductive substrates forming monomolecular thin layers (electrochemical studies, mercury drop junctions, conductive atomic force microscopy junctions). ${ }^{9}$ These methods are already quite popular, giving valuable information on the charge transport mechanisms at the molecular level. Nevertheless, they require relatively long preparation procedures, which are not always suitable for the integration of these materials in functional devices. Here we aimed to explore the possibility of employing a simple preparation 
procedure based on self-assembly approach, for which the supramolecular polymers are formed in solution, in order to carry out an investigation of their electrical characteristics at the "material" level.

The most popular conductive coordination polymers consists of stacks of conjugated porphyrins linked by organic ligand such as pyrazine or $4,4^{\prime}$-bipyridine. ${ }^{2 a}, 10$ The conductivity of these $1 \mathrm{D}$ coordination polymers may depend on the interaction of the metal $d$-orbitals with the $\pi^{*}$ level of the bridging ligand, ${ }^{4 \mathrm{~d}}$ although conductive properties ascribed to $\pi-\pi$ orbital overlap of the organic ligands have also been reported for complexes in which layers are formed by stacking of macrocyclic moietiess. ${ }^{11}$ We have recently shown that high conductivity in terpyridine-based coordination oligomers with a controlled length can be achieved, with charge transport most likely occurring via hopping. ${ }^{9 b}$ In that case the oligomeric wires were chemisorbed edge-on onto conductive substrates and the metal coated tip of conductive atomic force microscopy (AFM) was used as counter electrode in order to measure the transport along the wire.

Here we investigate the electrical properties of similar yet polymeric compounds for which both $\mathrm{d}-\pi$ and $\pi-\pi$ interactions may be expected, as potential functional materials, spontaneously assembled via physisorption on solid substrates. We have focused on coordination polymers obtained through combinations of the fully conjugated 4'-[10-[2-(4-pyridinyl)ethynyl]-9anthracenyl]-2,2':6',2"-terpyridine (TAP) organic tecton and $\mathrm{Pd}(\mathrm{II})$ or $\mathrm{Co}(\mathrm{II})$ as metallic nodes. The TAP tecton, bearing a monodentate pyridyl (py) and a tridentate 2,2':6',2''-terpyridine (terpy) divergently oriented coordinating poles, leads in the presence of metal ions to coordination polymers. ${ }^{12}$ Furthermore, the presence of an anthracene moiety connecting the terpyridyl and the pyridyl units through two ethynyl spacers confers high conformational rigidity and an inline arrangement of the two coordinating poles. ${ }^{12}$ Aiming at the construction of a 1D coordination polymer, the selection of a proper metal ion is crucial: ${ }^{13}$ one may indeed envisage the use of octahedral dicationic metals associated with two coordinating anions occupying the 
apical positions, ${ }^{14}$ or the use of square-planar metal centers. ${ }^{15}$ Both strategies have been explored, by using $\mathrm{Co}(\mathrm{II})$ and $\mathrm{Pd}(\mathrm{II})$ metal cations, respectively. The deposition of a solution containing the Co(II) or Pd(II) cations and TAP tecton on highly oriented pyrolitic graphite (HOPG) led to the formation, at the solid/liquid interface, of 1D structures which were imaged in situ by STM. ${ }^{12}$ The neutral complex formed by combining the TAP tecton with $\mathrm{CoCl}_{2}$ led to straight nanostructures up to $400 \mathrm{~nm}$ long, which appeared in STM images as aligned and regularly spaced bright features corresponding to the Co(II) ions. These spots were not laterally correlated, but grew either as single anisotropic nano-objects or as tightly packed parallel wires separated by a variable distance. Differently, the use of the Pd(II) cation, by design, yields a polycationic 1D network. Indeed, because of the dicationic nature of palladium center, its binding by the neutral ligand generates a charged square-planar assembling node formed by the palladium center and one terpyridine and one pyridine unit belonging to consecutive tectons. ${ }^{12,16}$ STM images showed in this case that the straight, parallel 1D wires obtained on graphite, were laterally correlated, because of the lateral intercalation of the counter anions leading thus to an overall a $2 \mathrm{D}$ neutral supramolecular array. ${ }^{12}$ Because of the strong affinity for the graphite surface, molecule-substrate interactions play a key role in the assembly process and in the formation of linearly correlated structures. In the present work spontaneous molecular assembly is obtained in solution aiming to investigate the effect of different metal ions on the structural, and functional properties of similar coordination polymers. 

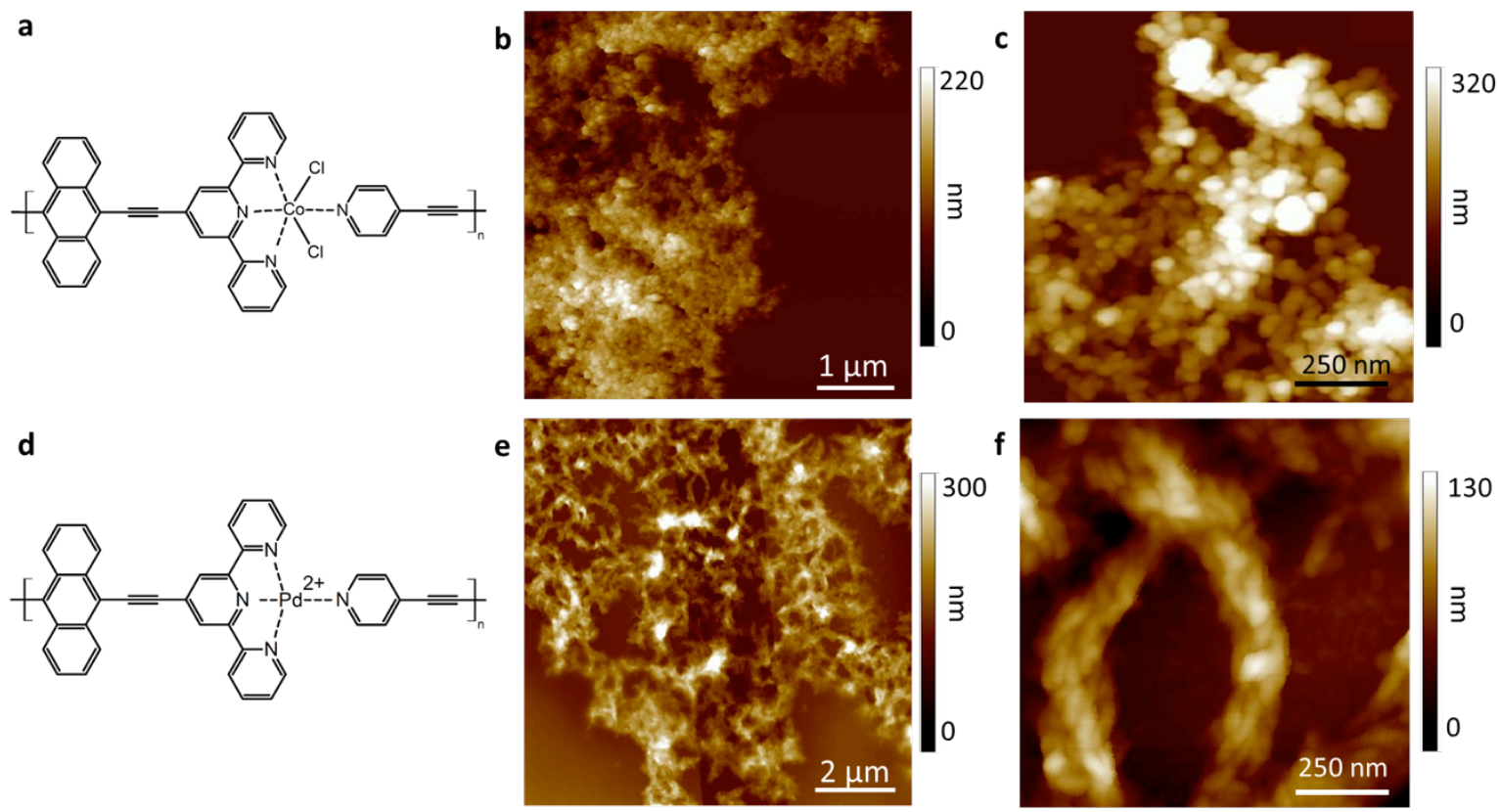

Figure 1: Chemical structures and AFM morphological characterization of (a-c) (CoTAP) $)_{n}$ and (d-f) (PdTAP $)_{n}$ self-assembled structures of coordination polymers deposited by drop casting onto $\mathrm{SiO}_{2}$ substrates.

\section{Results and discussions}

The two coordination polymers are formed in solution (see Methods) and successively deposited on solid substrates into thin films. Upon complexation a suspension is formed due to the low solubility of the coordination polymers, so that the transfer to the solid surface is similar to the deposition of aggregate structures obtained by solvent induced precipitation (SIP) methods. ${ }^{17}$ The morphologies of the two coordination polymers appear markedly different: differently from previously reported case, ${ }^{12}$ since the coordination polymers are formed in solution and not assembled at the solid/liquid interface, the effect of the molecule-substrate interactions is negligible in this case. Therefore we expect intermolecular forces to play a major role in the self- 
assembly process of these coordination polymers macroscopic structures. The optical micrographs of the $(\mathrm{CoTAP})_{\mathrm{n}}$ and $(\mathrm{PdTAP})_{\mathrm{n}}$ polymers already show a remarkable difference between the two films deposited onto $\mathrm{SiO}_{2}$ surfaces: the $\mathrm{Pd}(\mathrm{II})$ containing polymer displays micro-sized fibrillar features, whereas the Co(II) based compounds aggregate into grain-like structures (Figure S1). A more detailed morphological analysis carried out by AFM showed that the film composed of $(\mathrm{CoTAP})_{\mathrm{n}}$ consists of small globular aggregates with a diameter of ca. 10 $50 \mathrm{~nm}$ which are randomly spread over the substrate, (Figure $1 \mathrm{~b}, \mathrm{c})$ whereas the $(\operatorname{PdTAP})_{\mathrm{n}}$ film is composed by interconnected fibrillar structures forming networks (Figure $1 \mathrm{e}, \mathrm{f}$ ).

This markedly different morphology can be explained by considering the coordination geometry of the metal centers. In particular, since each TAP tecton bears two divergently oriented monodentate and tridentate sites and each $\mathrm{Co}(\mathrm{II})$ center behaves as a four connecting node, a 1D coordination polymer is generated through binding of $\mathrm{Co}(\mathrm{II})$ cations by four pyridyl units belonging to the terpy and py units of neighboring TAPs. For this coordination network, the presence of two $\mathrm{Cl}^{-}$anions in the axial positions leads to the octahedral coordination geometry around $\mathrm{Co}(\mathrm{II})$ cation. This geometry hampers an intermolecular packing which can be instead favored when a planar architecture such as the one of (PdTAP $)_{n}$ is formed, the later relying on the use of the tetracoordinated $\mathrm{Pd}(\mathrm{II})$ possessing a planar geometry to promote the generation of more packed structures (see supramolecular motifs in Figure 1). $\pi-\pi$ interactions can indeed play an important role in controlling the packing or assembly of organic molecules, also in metal coordinated compounds. In many structural descriptions of metal-ligand complexes $\pi-\pi$ stacking is invoked as a motif, where the most common $\pi$ interaction is an offset or slipped stacking, i.e. the rings are parallel displaced. ${ }^{18}$

This hypothesis was confirmed by the Grazing Incidence X-ray Diffraction (GIXRD) analysis of the two films deposited on $\mathrm{SiO}_{2}$ substrates (Figure 2). The 2D-GIXRD image recorded for the $(\mathrm{CoTAP})_{\mathrm{n}}$ film shows uniform rings, indicating that the film is formed by randomly oriented 
crystalline domains, of an average sizes in the range of $90-200 \AA$ (as shown in Table S1), in good accordance with AFM observations. From the radial integration of the diffraction pattern, four main periodicities ( $d$-spacings) are detected: $20.3 \AA, 10.8 \AA, 8 \AA$ and $3.4 \AA$, the former one being ascribed to the repetition of the monomer length, and the latter one to the $\pi$ - $\pi$ interactions between single monomers. Differently from the Co(II) polymer, the diffraction image of the $(\text { PdTAP) })_{n}$ film (Figure 2c) contains arc shaped Bragg spots symmetrical with respect to the surface normal, typical for highly ordered film in the out-of-plane direction but isotropic in the inplane (i.e. a cylindrical textured film). For this particular type of symmetry it is useful to analyse the scattering profile along the specular direction $\left(\mathrm{q}_{\mathrm{xy}} \sim 0\right)$ and along the in-plane direction $\left(\mathrm{q}_{\mathrm{z}} \sim 0\right)$, which in this case suggests that the majority of (PdTAP $)_{n}$ crystallites adopt an edge-on packing on the $\mathrm{SiO}_{2}$ substrate. Indeed, the intense Bragg spot along $\mathrm{q}_{\mathrm{z}}$ corresponds to a periodicity of $12 \AA$ between lattice planes parallel to the surface and can be related to the lamellae stacking, whereas along the $\mathrm{q}_{\mathrm{xy}}$ direction (Figure $2 \mathrm{e}$ ) two periodicities of $3.4 \AA$ and $9 \AA$ are clearly detected. The former periodicity is ascribed to the $\pi-\pi$ interaction between the anthracene moieties, whereas the latter could be attributed to the repetition of half of the chain backbone. The typical dimensions of the crystalline domains giving rise to these reflections are about $50 \AA$ except in the case of the $\pi$ - $\pi$ reflection for which the domains are slightly smaller (about $40 \AA$ ). 

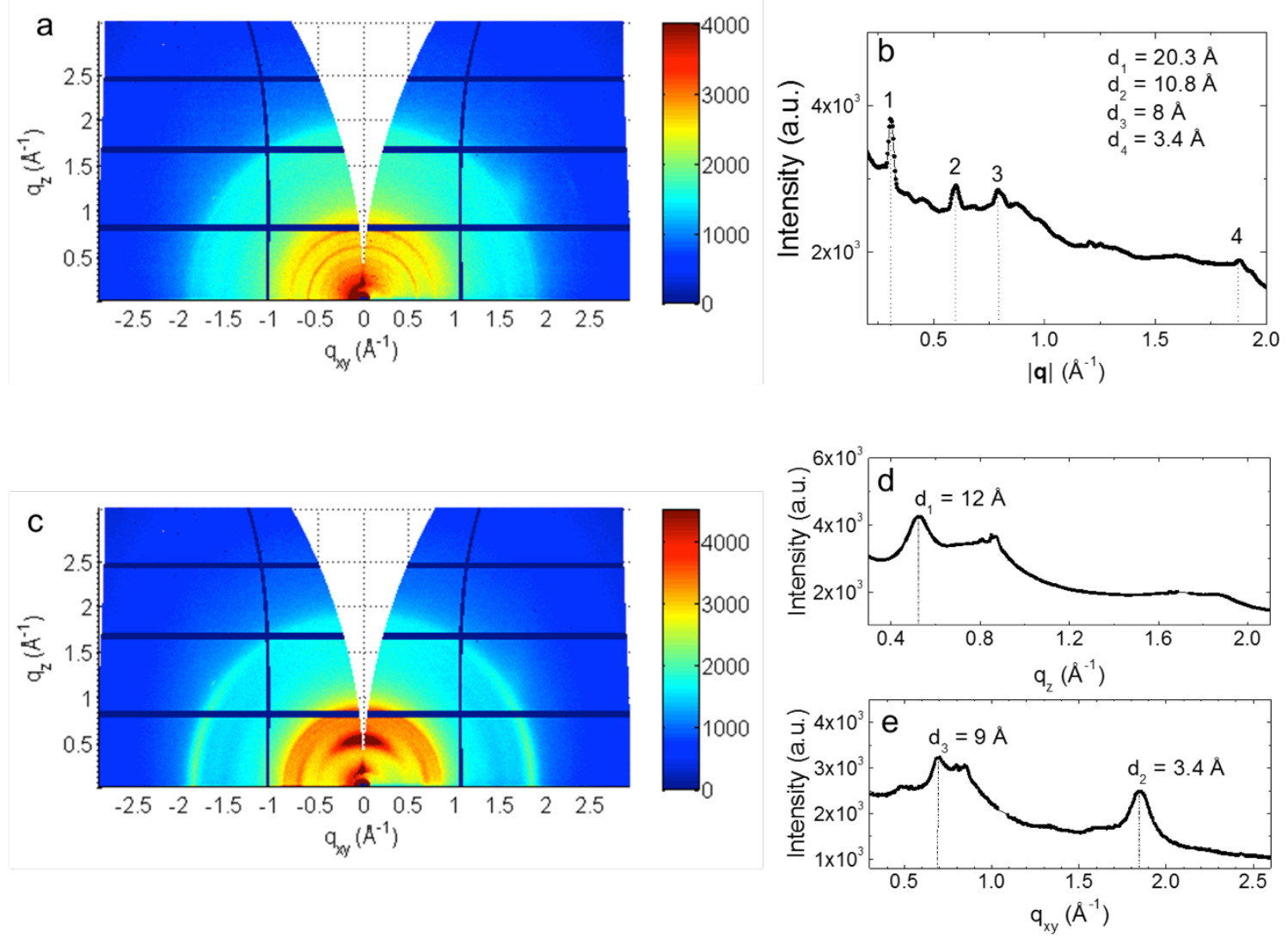

Figure 2: GIXRD analysis of the $(\operatorname{CoTAP})_{\mathrm{n}}(\mathrm{a}, \mathrm{b})$ and $(\operatorname{PdTAP})_{\mathrm{n}}(\mathrm{c}-\mathrm{e})$ films. a, c) 2D-GIXRD images; b) Radial integration of the 2D-GIXRD image in a). d, e) Scattering intensity integrated along the specular $\left(\mathrm{q}_{\mathrm{xy}} \sim 0\right)$ (d) and in-plane (e) directions. The most relevant peaks are highlighted and the corresponding $d$-spacing are indicated.

The molecular packing of the (PdTAP $)_{n}$ film was estimated by performing simulations of the diffraction patterns. The monomer shown in Figure 3 a was used as repeating unit. The chosen unit cell consisted of four monomers and eight acetate counter ions, to balance the positive charge of each $\mathrm{Pd}(\mathrm{II})$ metal center (Figure $3 \mathrm{~b}$ ). Consecutive chains in the stack point in opposite directions to balance the dipole moment, as indicated by green and red arrows. The 'head-to-tail' 
orientation of stacked pyridyl groups is indeed energetically more favorable than the 'head-tohead' one. ${ }^{19}$
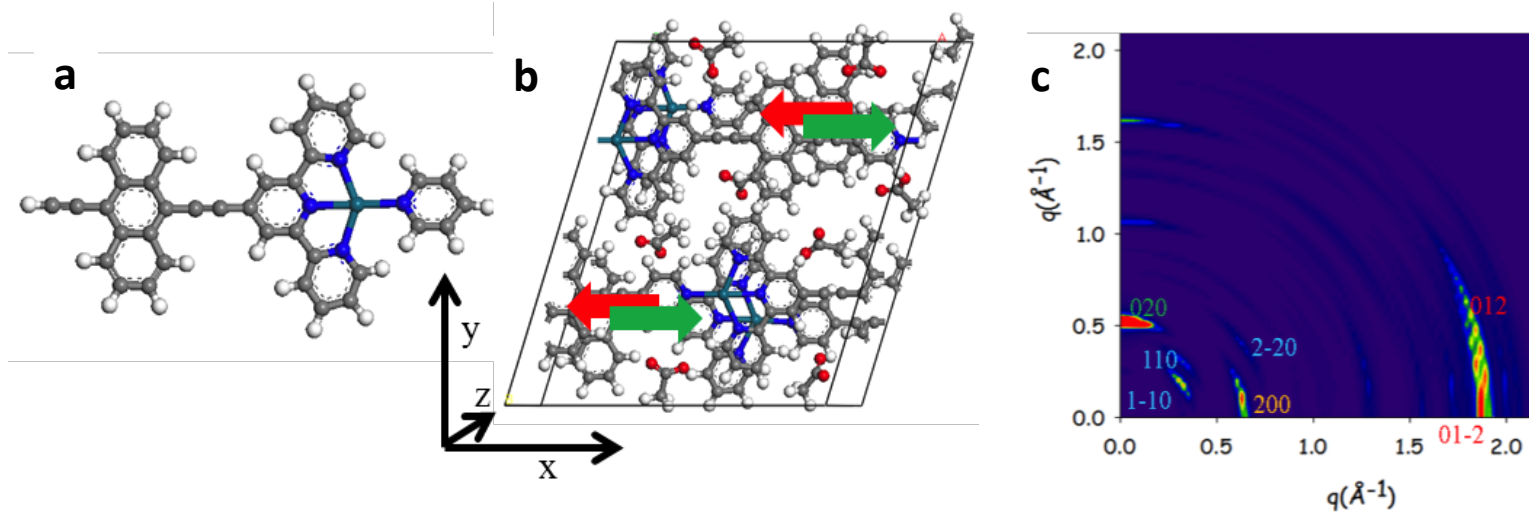

Figure 3: a) Structure of the monomer used for the packing. b) Unit cell adopted for the simulations (green: Pd, blue: N, red: O, grey: C, white: H). c) Simulated diffraction pattern of the PdTAT film, corresponding to the packing in $b$, being the $x z$ plane the substrate surface. Numbers refer to the different Miller indexes.

From the simulations it is clear that polymer chains arranged perpendicularly or stacked face-on onto the surfaces are not described by the experimental diffraction pattern (Figure S8, S9). The simulated pattern in Figure 3c, matching instead the experimental one, is the result of an edge-on molecular configuration where the monomers organize with their short axes perpendicularly to the surface ( $x z$ plane in Figure 3b). In the cell, the monomers are shifted by $2 \AA$ with respect to each other along the lamellae direction ( 010 direction, $y$ axis) and by $4 \AA$ along the amol direction ( $x$ axis). The Miller index marking the peaks in Figure $3 \mathrm{c}$ are associated with the different planes directions: the 020 index refers to the lamellae direction ( $y$ axis; the shortest distance in between two polymer chains is about $2.6 \AA$ ); the 200 index is along the molecular axis ( $x$ axis, corresponding to half of the monomer length); the 012 and $01-2$ indexes refer to the $\pi$-stacking direction ( $z$ axis) and the peaks associated to the 110/1-10/2-20 indexes are mainly due to counter 
ions. The calculated lamellae direction spacing amounts to $11.49 \AA$, the spacing along the molecular direction corresponds to $9.74 \AA$ and that in the $\pi$-stacking direction is $3.36 \AA$. These data are in very good agreement with the corresponding experimental values of 12,9 and $3.4 \AA$ for the lamellae, molecular length and $\pi$-stacking directions, respectively. It is worth noting that the diffraction pattern above is not very sensitive to small changes in geometry of the system: when shifting the two polymer chains towards a perfect co-facial orientation, no significant changes have been detected in the simulated patterns.

This structural characterization clearly shows markedly different supramolecular organizations of the two coordination polymers, with a longer-range order and more distinct $\pi-\pi$ aggregation for the planar (PdTAP) $)_{\mathrm{n}}$ polymer.

The electrical characterization was performed on thin films prepared by drop casting a few $\mu \mathrm{L}$ of the suspension containing the $(\mathrm{CoTAP})_{\mathrm{n}}$ or the $(\mathrm{PdTAP})_{\mathrm{n}}$ polymers on $\mathrm{SiO}_{2}$ substrates exposing pre-patterned Au electrodes having interelectrode gaps of 2.5 to $10 \mu \mathrm{m}$. Two probe measurements revealed conductivity only for devices integrating $(\mathrm{PdTAP})_{\mathrm{n}}$ as electroactive layer. In particular, currents of the order of $\mu \mathrm{A}$ with a linear behavior in the range of $\pm 10 \mathrm{~V}$ were observed for the $\mathrm{Pd}$ polymer, whereas the current was always below the detection limit, i.e. in the pA range, for the $(\mathrm{CoTAP})_{\mathrm{n}}$ film (Figure $\left.4 \mathrm{a}\right)$. Because of the insoluble nature of the coordination polymers, extremely inhomogeneous films (thus surface coverage) in between the electrodes were obtained, consequently a quantitative assessment of the conductivity of these materials remains challenging.

Conductivity measurements of the (PdTAP $)_{n}$ complex at the nanoscale were performed by $\mathrm{C}$ AFM in a vertical configuration using a gold electrode patterned on silicon oxide substrate. The conductive $\mathrm{Pt} / \mathrm{Ir}$ tip was scanned over the surface of the film and the voltage was varied during the same scan, as marked in the current maps of Figure 4c. By comparing the topography and 
current maps it is possible to observe the response in terms of current modulation with the bias applied between the gold electrode and the C-AFM tip. The average currents detected in the bias range of $\pm 10 \mathrm{~V}$ are on the order of a few $\mathrm{pA}$ : the average currents at $+5 \mathrm{~V}$ and $-5 \mathrm{~V}$ amount to 0.40 and $0.42 \mathrm{pA}$ (see the current map in Figure S12), respectively, which indicate that there is not preferential charge injection from one electrode (C-AFM tip) with respect to the other (gold substrate), probably because of the similar electrodes work functions $(\sim 5 \mathrm{eV})$. Such low current values are very common in TR-TUNA C-AFM experiments. ${ }^{20}$ Noteworthy, a current is clearly detected on the films hundreds nm away from the biased substrate, which suggests efficient pathways for charge transport through the thick film. By comparing the current maps at a fixed bias with the topography images, no clear scaling of the current with the thickness of the film is visible, which may be an indication of contact limited junction. However, as it can also be observed by the comparison of the height section with the current profile in Figure $4 \mathrm{~d}$, when the tip encounters a topographical protrusion during the lateral scan, a sudden increase in the current signal is recorded. Such feature can likely be ascribed to a variation of the actual contact area. 

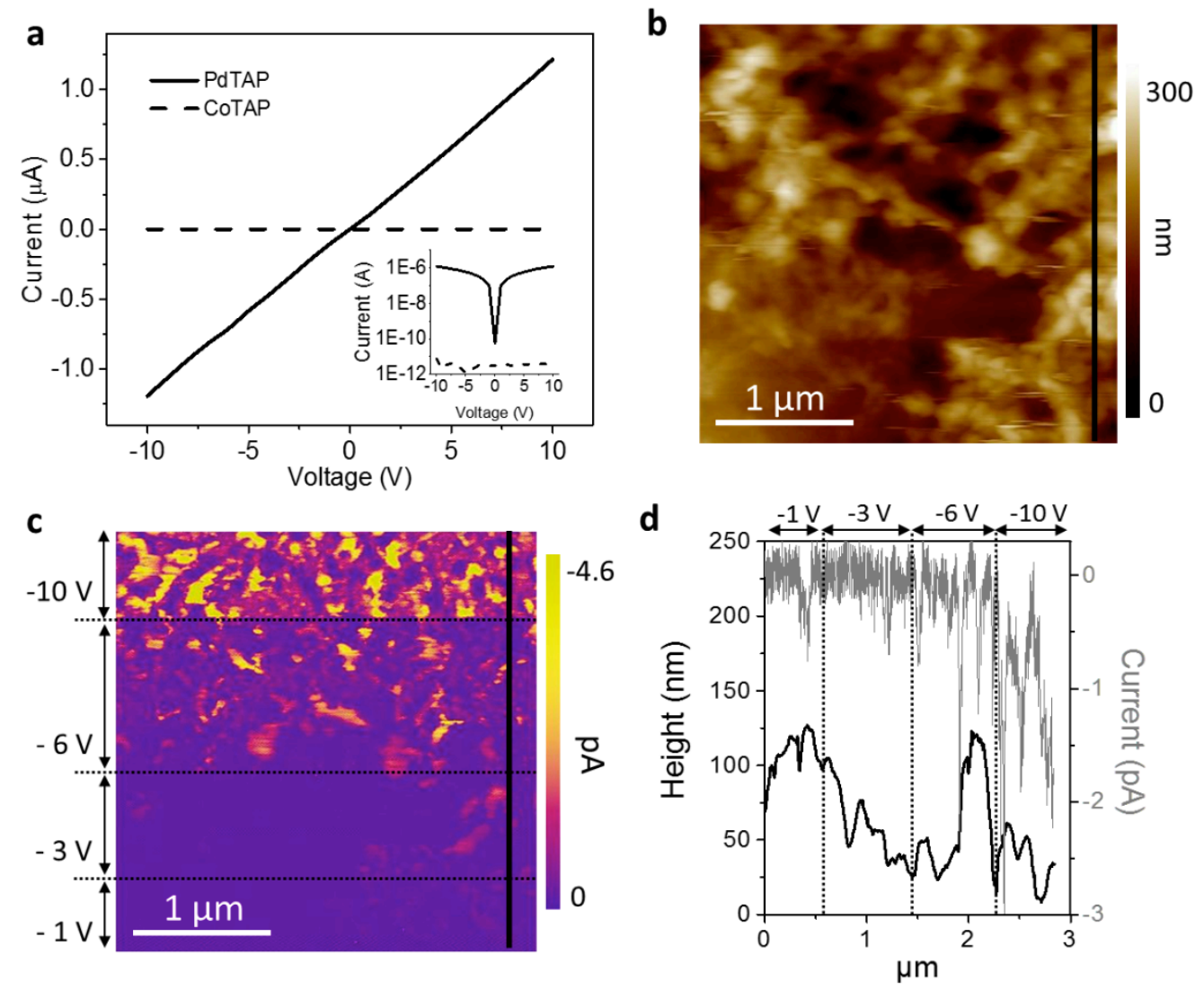

Figure 4. Electrical characterization of the (CoTAP $)_{n}$ and (PdTAP) $)_{n}$ polymers. (a) Currentvoltage measurements performed in a two electrodes configuration $\left(\mathrm{SiO}_{2}\right.$ substrates with prepatterned interdigitated gold electrodes, inter-electrode gap $\mathrm{L}=10 \mu \mathrm{m}$, electrodes length $\mathrm{W}=10$ mm). (b-d) Nanoscale C-AFM characterization of (PdTAP) $)_{n}$ film on a gold electrode: (b) topography and (c) current map images. In the current map in (c) four different areas are visible where the bias applied to the bottom electrode is increased from $-1 \mathrm{~V}$ to $-10 \mathrm{~V}$. (d) Height (black) and current (grey) profiles along the vertical black lines marked in (b, c).

As mentioned above, electron transfer processes taking place between adjacent molecules via two possible pathways, i.e. metal- $\pi$ and/or $\pi$ - $\pi$ interactions, have been reported for other transitionmetal coordinated systems in literature. ${ }^{21}$ The presence of strong aromatic units favoring the 
assembly of coordination polymers into supramolecular structures by $\pi$ - $\pi$ interactions was demonstrated in many cases to be a supplementary requirement for the conductivity of this type of compounds. ${ }^{22}$ In the present case, it is likely that $\pi-\pi$ inter-chain interactions play an important role in this respect. Below we report the results of theoretical calculations aiming at assessing intra-chain and inter-chain transport in the $(\mathrm{PdTAP})_{\mathrm{n}}$ polymer.

In order to account properly for the +2 oxidation state of the metal center, two acetate counter ions acting as the outer coordination shell were flanked around the $\mathrm{Pd}(\mathrm{II})$ atoms. Calculations performed at the B3LYP level for an infinite one-dimensional polymer (thus, with explicit counter ions) yield an electronic band gap of $2.31 \mathrm{eV}$, with the valence band (VB) lying at -5.17 $\mathrm{eV}$ and the conducting band (CB) at $-2.86 \mathrm{eV}$ at the $\Gamma$ point. Note that the calculated value for the energy of the VB top is in an excellent agreement with the experimental data $(-5.16 \pm 0.02 \mathrm{eV})$ obtained by photoelectron spectroscopy measurements in air (see Experimental section). The width of the valence (VB) and conduction (CB) bands of 0.001 and $0.05 \mathrm{eV}$, respectively, translates into very small effective transfer integrals between successive units along the polymer chains $\left(2.5 \times 10^{-4} \mathrm{eV}\right.$ for holes and $0.0125 \mathrm{eV}$ for electrons $)$. We thus anticipate limited intrachain charge transport properties. This is borne out by the analysis of the frontier crystal orbitals showing localization of the LUCO over the anthracene-pyridine segments and one of the terpyridine ring of the polymer repeating unit, while the $\mathrm{HOCO}$ is localized on the metal center and the remaining two terpyridyl rings, as depicted in Figure 5. Such a spatial confinement of the frontier orbitals suggests weak electronic communications along the polymer backbones for both holes and electrons. Note that these calculations were repeated for different conformations of the repeating units, namely different torsion angles between successive terpyridyl, pyridyl and anthracene groups, and consistently result in similar values for the electronic bandwidth and band gap values, as detailed in SI. 
a

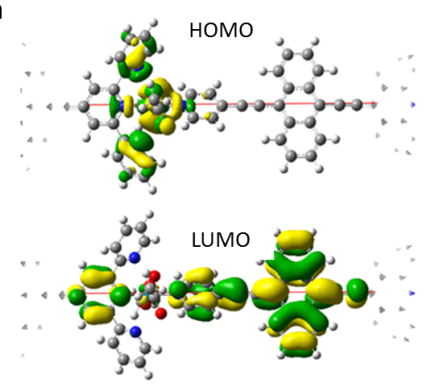

b

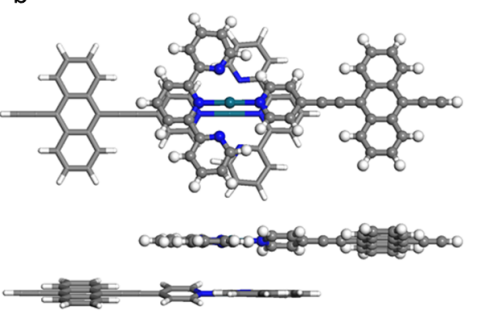

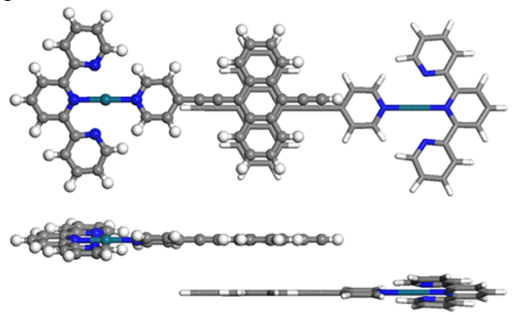

Figure 5. a) Shape of the frontier crystal orbitals. The red line refers to the translation vector. b, c) Top and lateral view representations of two overlapping monomer configurations considered for the electronic structure calculations: overlap of the metal centers (b) and of the anthracene groups of the ligand (c). For clarity, the two overlapping monomers in b, c are represented in different graphical models.

Although the diffraction spectrum is not sensitive to small displacements of single polymer chain with respect to its neighbor, we anticipate that inter-chain charge transport abilities (i.e. the charge transfer integrals) are very sensitive to even small $(\sim 0.1 \AA)$ shifts. Here, for simplicity and because we are merely interested in qualitative trends, we consider only a perfect co-facial orientation (in two different arrangements) for the calculation of the inter-chain electron transfer integrals. Also, since in the lamellae direction the distance between neighbor polymer backbones is large ( $\sim 5 \AA)$, we focus only on transport along the $\pi$-stacking direction.

Two different segments of the polymer backbones have been extracted from the packing in Figure 3b: the first with neighboring monomers overlapping through the Pd-Pd metal centers and the second with neighboring monomers overlapping through the anthracene groups of the ligands, as depicted in Figure 5b, c. We stress that both types of contact are consistent with the packing arrangement shown in Figure 4. In view of the confined character of the frontier MOs, the 
electronic couplings mediating hole and electron inter-chain diffusion have been computed for monomers. Here, for practical reasons, the two acetate counter ions are substituted with two negative (-1e) point charges, at a distance of $2 \AA$ from the metal center, in line with earlier experimental data obtained for a similar ligand. ${ }^{23}$

The inter-chain charge transport ability is assessed by calculating the effective electronic coupling using an in-house adiabatic projective method, as applied to DFT/B3LYP electronic structure calculations (see Experimental section for more details). The frontier orbitals obtained for the monomer are reported in Figure S13. Although compared to the explicit counter ions calculations the use of point charges leads to non-negligible changes in the absolute energies of the MOs, their shape and spatial (de)localization are hardly affected. Namely the molecular HOMO is mainly localized on the metal center and partly on the terpyridyl groups, while the LUMO is mainly localized on the anthracene and ligand pyridyl group. It is worth noting that a similar localization of the frontier orbitals is found for different configurations of the pyridyl group with respect to the molecular plane. As the electronic coupling primarily reflects wavefunction overlap, the model systems considered here are realistic.

The electronic coupling mediating charge transport in the $\pi$-stacking direction is very high for electrons, with $t_{\mathrm{e}}$ reaching up to $190 \mathrm{meV}$ when the anthracene groups are overlapping (Figure 5c). Likewise, a large and comparable $t_{\mathrm{h}}$ value of $\sim 180 \mathrm{meV}$ is obtained for holes but now when the metal centers overlap (Figure 5b). Hence, the electronic structure calculations suggest both high intermolecular hole and electron transport properties, mediated by Pd-Pd and anthraceneanthracene couplings, respectively.

\section{Conclusions}


In summary, we investigated the organization on surfaces of coordination polymers formed upon combining metal cations such as $\mathrm{Co}(\mathrm{II})$ or $\mathrm{Pd}(\mathrm{II})$ with an organic tecton based on an anthracene core bearing two ethynyl groups equipped with a pyridyl and a terpyridyl groups. We found that the formation of their supramolecular structures on $\mathrm{SiO}_{2}$ surface is governed by the coordination geometry of the metal centers. In particular, the planarity of squared Pd(II) based polymers allows for the formation of $\pi-\pi$ stacked fibrillar structures where crystalline domains have specific preferred orientations. The improved order of these molecular systems, with respect to the corresponding octahedral $\mathrm{Co}$ (II) polymer, where the $\pi$ - $\pi$ stacking is hindered, is most likely responsible for the completely different electrical behavior. The calculation of the electronic structure of the $\mathrm{Pd}(\mathrm{II})$ polymer yields hole and electron couplings that are both vanishingly small along the chains, because of the spatial localization of the frontier crystal orbitals over either the Pd- or the anthracene-mostly units. On the other hand, these couplings are predicted to be much larger (in the order of $0.2 \mathrm{eV}$ ) between adjacent chains, as a result of the favorable Pd-Pd and anthracene-anthracene wavefunction overlaps along the fiber direction. Both the macroscopic and the C-AFM electrical measurements revealed indeed promising charge transport properties for the $(\mathrm{PdTAP})_{\mathrm{n}}$ polymer. Nevertheless, the difficulty of processing of such a material did not allow, under the conditions used, the extraction of more quantitative information. In this respect, more effort needs to be addressed towards improvement of the film deposition procedures and towards fabrication of mesoscopically-defined structures of the coordination polymers. We believe that the presented results open new perspectives on the engineering of conductive coordination polymers towards their applications in functional devices.

\section{Experimental details:}


Cobalt chloride hexahydrate $\left(\mathrm{CoCl}_{2} \cdot 6 \mathrm{H}_{2} \mathrm{O}\right)$, palladium acetate tetrahydrate $\left(\mathrm{Pd}\left(\mathrm{CH}_{3} \mathrm{COO}\right)_{2} \cdot 4 \mathrm{H}_{2} \mathrm{O}\right)$, isopropanol (IPA) and chloroform $\left(\mathrm{CHCl}_{3}\right)$ were purchased from Sigma Aldrich. The terpyridyl-anthracene-pyridyl (TAP) ligand was synthesized as previously described. ${ }^{21}$ The polymer preparation was carried out by mixing $1 \mathrm{~mL}$ of $1 \mathrm{mM}$ TAP $\mathrm{CHCl}_{3}$ solution with either $100 \mu \mathrm{L}$ of $10 \mathrm{mM}$ of $\mathrm{CoCl}_{2} \cdot 6 \mathrm{H}_{2} \mathrm{O}$ solution in IPA or $100 \mu \mathrm{L}$ of $10 \mathrm{mM}$ $\mathrm{Pd}\left(\mathrm{CH}_{3} \mathrm{COO}\right)_{2} \cdot 4 \mathrm{H}_{2} \mathrm{O}$ solution in $\mathrm{CHCl}_{3}$, that is by mixing them a $1: 1$ ratio. Suspension in chloroform was obtained which was then drop cast on the silicon oxide support. Films were prepared by drop casting few $\mu \mathrm{L}$ of the dispersion in $\mathrm{CHCl}_{3}$ on the solid substrates.

Photophysical Characterization. Bright-field, dark-field, fluorescence and polarized light images were taken with an Olympus BX51 optical microscope (100X objective) equipped with an X-Cite series 120 fluorescence cube 37088 U-MWBS3. Fluorescence microscopy images were taken on an Olympus BX51 microscope (100X objective) at $\lambda_{\mathrm{ex}}=450-480 \mathrm{~nm}$ and $\lambda_{\mathrm{em}}>515 \mathrm{~nm}$. Absorption spectra were recorded with a JASCO spectrophotometer - V670, on air equilibrated $\mathrm{CHCl}_{3}$ (Uvasol solvent, Merck Millipore) solutions at room temperature (ca. $20^{\circ} \mathrm{C}$ ), with concentrations ranging from $1 \times 10^{-5}$ to $5 \times 10^{-4} \mathrm{M}$. Solution were examined in $1-\mathrm{cm}$ and $1-\mathrm{mm}$ spectrofluorimetric quartz cells (Hellma). The fluorescence spectra were recorded by using a Fluorolog 3 spectrofluorimeter (Jobin-Yvon). For Uv-Vis titration, stock solutions at $1 \times 10^{-3} \mathrm{M}$ of $\mathrm{CoCl}_{2}$ and $\mathrm{Pd}(\mathrm{OAc})_{2}$ were used.

Grazing Incidence X-ray Diffraction (GIXRD) measurements were performed at XRD1 beamline of ELETTRA Synchrotron facility in Trieste (Italy) using a monochromatic beam of energy E = $12.4 \mathrm{keV}$, (corresponding to a wavelength, $\lambda$, of $1 \AA$ ) and size $200 \times 200 \mu \mathrm{m}^{2}$. The incident angle of the X-ray beam, $\alpha_{\mathrm{i}}$, was chosen just above the critical angle for total reflection of the organic film (i.e. $0.1^{\circ}$ ). The diffraction patterns were collected by a 2D camera (Pilatus detector) placed normal to the incident beam direction at a distance of $250 \mathrm{~mm}$ from the sample. All the results are 
expressed in terms of scattering vector $\mathbf{q}$ defined as $q=\frac{4 \pi}{\lambda} \sin (\theta)$, where $\theta$ is half of the scattering angle.

Atomic Force Microscopy (AFM) morphological characterization was performed in tapping mode in a Dimension 3100 microscope equipped with a Nanoscope IV controller (Digital Instruments). Commercial silicon cantilevers with a nominal spring constant of $40 \mathrm{~N} / \mathrm{m}$ were used for morphological characterization in tapping mode.

Conductive atomic force microscopy (C-AFM) experiments were performed in torsion-resonance tunneling current (TR-TUNA C-AFM) mode in a Multimode V (Veeco) microscope equipped with a Nanoscope V controller using Pt/Ir-coated Si tips with $225 \mu \mathrm{m}$ long cantilever, nominal tip radius of $20 \mathrm{~nm}$ and spring constant in the range $0.5-9.5 \mathrm{~N} / \mathrm{m}$. The torsion amplitude was used as the feedback signal to measure surface morphology. The current maps were obtained by biasing a bottom gold electrode and mapping the current of the material on top of the gold.

Gold electrodes patterned on $\mathrm{SiO}_{2}$ (Fraunhofer Institute) substrates were cleaned by subsequent ultrasonication bath in acetone and isopropanol prior to use. A Keithley 2636A source meter was for the two probe current-voltage characteristics measurements under controlled atmosphere. Two terminal bottom contacts devices were prepared by drop casting the complex solution onto patterned substrates.

The work function of $\mathrm{Au}$ substrates and the ionization potential of the different coordination polymer based films were measured by means of ambient photoelectron spectroscopy with a Riken Keiki spectrophotometer (Japan) model AC-2. The conditions used during the measurements were scanning energies in the range of 4.0 to $6.2 \mathrm{eV}$ with a measurement interval of $0.05 \mathrm{eV}$ and a UV spot intensity of $35-50 \mathrm{nW}$, to warrant a $20 \mathrm{meV}$ accuracy. 
The packing of the system has been analyzed with the Material Studio v6.0.0 program (Accelrys Software Inc, San Diego, CA, 2011) with the Forcite module to optimize the structure at the molecular mechanics (MM) level with pcff Force Field. ${ }^{24}$ Following, Molecular Dynamic (MD) calculations have been performed at two temperatures and time scales; the first MD run at $600 \mathrm{~K}$ for $3 \mathrm{~ns}$ has been followed by a shorter (1ns) run at room temperature (RT). This procedure has been repeated until stable @RT crystal structures have been obtained. The GIWAXS (synchrotron wavelength of $1 \AA$ ) patterns of these stable structures have then been compared with the experimental data.

The ab-initio calculations have been performed using the GAUSSIAN09 software $^{25}$ with periodic boundary conditions (PBC) with a monomer considered as a repeating unit of the coordination polymer and two acetate counterions. The ligand's structure has been optimized at the DFT level, using the B3LYP functional ${ }^{26}$ and the $6-31 \mathrm{G}(\mathrm{d})$ basis set. ${ }^{27}$ For the metal center the LANL2DZ basis set has been used, 28 as it consists of an effective core potential for heavy atoms. The intrachain hole and electron charge transfer properties has been then assessed as W/4 estimation of an infinite polymer chain coupling, where $\mathrm{W}$ is the bandwidth of the valence and conducting band, respectively.

The inter-chain charge transport has been estimated by calculating charge transfer integrals with the adiabatic projective method for calculation of transfer integrals ${ }^{29}$ for $\pi$-stacked dimers of monomers extracted from the crystal systems using the GAUSSIAN09 software. The system is divided into fragments, in which an electron or hole is localized on a fragment, and can hop from one fragment to another. ${ }^{30}$ The fragment calculations, performed by using DFT method (B3LYP functional with the $6-31 \mathrm{G}(\mathrm{d}, \mathrm{p})$ basis set), follow a procedure where the orbitals of a pair of molecules (a dimer) are projected onto a basis set defined by the orbitals of each individual molecule (the fragments). The obtained set of orthogonal molecular orbitals energies of the dimer are then used to rewrite the Fock matrix in the new localized basis set, to obtain a block-diagonal 
matrix. The main advantage of this method is the possibility to analyze pairs of different molecules, since it is not necessary to assume that the energies of the frontier orbitals of the two fragments are equal.

\section{Acknowledgements}

This work was financially supported by University of Strasbourg, CNRS, Istitut Universitaire de France (IUF), the ERC project SUPRAFUNCTION (GA-257305), the EC Marie-Curie projects ITN iSwitch (GA no. 642196) and IEF GALACTIC (PIEF-GA-2013-628563), the Agence Nationale de la Recherche through the LabEx project Chemistry of Complex Systems (ANR-10-LABX-0026_CSC), and the International Center for Frontier Research in Chemistry (icFRC). DB is a FNRS Research Director.

\section{References}

1. Y. Yan and J. B. Huang, Coord. Chem. Rev., 2010, 254, 1072-1080.

2. a) A. Y. Robin and K. M. Fromm, Coord. Chem. Rev., 2006, 250, 2127-2157; b) D. Heim, D. Ecija, K. Seutert, W. Auwarter, C. Aurisicchio, C. Fabbro, D. Bonifazi and J. V. Barth, J. Am. Chem. Soc., 2010, 132, 6783-6790; c) D. Ecija, S. Vijayaraghavan, W. Auwarter, S. Joshi, K. Seufert, C. Aurisicchio, D. Bonifazi and J. V. Barth, Acs Nano, 2012, 6, 4258-4265; d) S. De Feyter, M. M. S. Abdel-Mottaleb, N. Schuurmans, B. J. V. Verkuijl, J. H. van Esch, B. L. Feringa and F. C. De Schryver, Chem. Eur. J., 2004, 10, 1124-1132; e) T. Bauer, Z. K. Zheng, A. Renn, R. Enning, A. Stemmer, J. Sakamoto and A. D. Schlüter, Angew. Chem. Int. Ed., 2011, 50, 7879-7884; f) J. X. Liu, B. Lukose, 0. Shekhah, H. K. Arslan, P. Weidler, H. Gliemann, S. Brase, S. Grosjean, A. Godt, X. L. Feng, K. Müllen, I. B. Magdau, T. Heine and C. Wöll, Sci. Rep., 2012, 2, 921.

3. H. Furukawa, K. E. Cordova, M. O'Keeffe and O. M. Yaghi, Science, 2013, 341, 1230444-1230456.

4. a) G. Givaja, P. Amo-Ochoa, C. J. Gomez-Garcia and F. Zamora, Chem. Soc. Rev., 2012, 41, 115-147; b) J. Gómez-Herrero and F. Zamora, Adv. Mater., 2011, 23, 5311-5317; c) B. Hu, J. Geng, L. Zhang and W. Huang, Sol. St. Chem., 2014, 215, 102-108; d) C. Janiak, Dalton Trans., 2003, 2781-2804; e) Z. T. Xu, Coord. Chem. Rev., 2006, 250, 2745-2757; f) D. Gentili, G. Givaja, R. Mas-Balleste, M. R. Azani, A. Shehu, F. Leonardi, E. Mateo-Marti, P. Greco, F. Zamora and M. Cavallini, Chem. Sci., 2012, 3, 2047-2051.

5. D. Sheberla, L. Sun, M. A. Blood-Forsythe, S. Er, C. R. Wade, C. K. Brozek, A. AspuruGuzik and M. Dinca, J. Am. Chem. Soc., 2014, 136, 8859-8862.

6. Y. M. Sun, P. Sheng, C. A. Di, F. Jiao, W. Xu, D. Qiu and D. B. Zhu, Adv. Mater., 2012, 24, 932-937. 
7. a) L. De Cola and P. Belser, Coord. Chem. Rev., 1998, 177, 301-346; b) A. Wild, A. Winter, F. Schlutter and U. S. Schubert, Chem. Soc. Rev., 2011, 40, 1459-1511.

8. J. Park, A. N. Pasupathy, J. I. Goldsmith, C. Chang, Y. Yaish, J. R. Petta, M. Rinkoski, J. P. Sethna, H. D. Abruña, P. L. McEuen and D. C. Ralph, Nature, 2002, 417, 722-725.

9. a) T. Kurita, Y. Nishimori, F. Toshimitsu, S. Muratsugu, S. Kume and H. Nishihara, J. Am. Chem. Soc., 2010, 132, 4524-4525; b) C. Musumeci, G. Zappala, N. Martsinovich, E. Orgiu, S. Schuster, S. Quici, M. Zharnikov, A. Troisi, A. Licciardello and P. Samorì, Adv. Mater., 2014, 26, 1688-1693; c) K. Terada, H. Nakamura, K. Kanaizuka, M. Haga, Y. Asai and T. Ishida, Acs Nano, 2012, 6, 1988-1999; d) N. Tuccitto, V. Ferri, M.

Cavazzini, S. Quici, G. Zhavnerko, A. Licciardello and M. A. Rampi, Nat. Mater., 2009, 8, 41-46.

10. M. Hanack and M. Lang, Adv. Mater., 1994, 6, 819-833.

11. a) C. H. Hendon, D. Tiana and A. Walsh, Phys. Chem. Chem. Phys., 2012, 14, 13120 13132; b) Z. A. Bao, A. J. Lovinger and J. Brown, J. Am. Chem. Soc., 1998, 120, $207-$ 208; c) W. Zhang, K. Ochi, M. Fujiki, M. Naito, M. Ishikawa, K. Kaneto, W. Takashima, A. Saeki and S. Seki, Adv. Funct. Mater., 2010, 20, 3941-3947.

12. M. Surin, P. Samorì, A. Jouaiti, N. Kyritsakas and M. W. Hosseini, Angew. Chem. Int. Ed., 2007, 46, 245-249.

13. R. Dobrawa, M. Lysetska, P. Ballester, M. Grüne and F. Würthner, Macromolecules, $2005,38,1315-1325$.

14. a) A. Jouaiti, M. W. Hosseini and N. Kyritsakas, Chem. Commun., 2002, 1898-1899; b) A. Jouaiti, V. Jullien, M. W. Hosseini, J. M. Planeix and A. De Cian, Chem. Commun., 2001, 1114-1115.

15. I. Eryazici, C. N. Moorefield and G. R. Newkome, Chem. Rev., 2008, 108, 1834-1895.

16. J. E. Beves, E. C. Constable, C. E. Housecroft, M. Neuburger and S. Schaffner, Inorg. Chem. Commun., 2007, 10, 1185-1188.

17. M. El Gemayel, M. Treier, C. Musumeci, C. Li, K. Müllen and P. Samorì, J. Am. Chem. Soc., 2012, 134, 2429-2433.

18. C. Janiak, J. Chem. Soc., Dalton Trans., 2000, 3885-3896.

19. A. N. Khlobystov, A. J. Blake, N. R. Champness, D. A. Lemenovskii, A. G. Majouga, N. V. Zyk and M. Schroder, Coord. Chem. Rev., 2001, 222, 155-192.

20. a) S. A. L. Weber and R. Berger, Appl. Phys. Lett., 2013, 102, 163105-163113; b) S. A. L. Weber, N. Haberkorn, P. Theato and R. Berger, Nano Lett., 2010, 10, 1194-1197.

21. S. L. Zheng, J. P. Zhang, W. T. Wong and X. M. Chen, J. Am. Chem. Soc., 2003, 125, 6882-6883.

22. a) M. Munakata, L. P. Wu, T. Kuroda-Sowa, M. Maekawa, Y. Suenaga, G. L. Ning and T. Kojima, J. Am. Chem. Soc., 1998, 120, 8610-8618; b) M. Munakata, G. L. Ning, Y. Suenaga, T. Kuroda-Sowa, M. Maekawa and T. Ohta, Angew. Chem. Int. Ed., 2000, 39, 4555-4557.

23. Ž. D. Bugarčić, B. Petrović and E. Zangrando, Inorg. Chim. Acta, 2004, 357, 26502656.

24. J. R. Hill and J. Sauer, J. Phys. Chem., 1995, 99, 9536-9550.

25. M. J. Frisch, G. W. Trucks, H. B. Schlegel, G. E. Scuseria, M. A. Robb, J. R. Cheeseman, G. Scalmani, V. Barone, B. Mennucci, G. A. Petersson, H. Nakatsuji, M. Caricato and H. P. H. X. Li, A. F. Izmaylov, J. Bloino, G. Zheng, J. L. Sonnenberg, M. Hada, M. T. Ehara, K. Toyota, R. Fukuda, J. Hasegawa, M. Ishida, T. Nakajima, Y. Honda, O. Kitao, H. Nakai, T. J. Vreven, J. E. Peralta, F. Ogliaro, M. Bearpark, J. J. Heyd, E. Brothers, K. N. Kudin, V. N. Staroverov, R. Kobayashi, J. Normand, K. Raghavachari, A. Rendell, J. C. Burant, S. S. Iyengar, J. Tomasi, M. Cossi, N. Rega, J. M. Millam, M. Klene, J. E. Knox, J. B. Cross, V. Bakken, C. Adamo, J. Jaramillo, R. Gomperts, R. E. Stratmann, O. Yazyev, A. J. Austin, 
R. Cammi, C. Pomelli, J. W. Ochterski, R. L. Martin, K. Morokuma, V. G. Zakrzewski, G. A. Voth, P. Salvador, J. J. Dannenberg, S. Dapprich, A. D. Daniels, Farkas, J. B. Foresman, J. V. Ortiz, J. Cioslowski and D. J. Fox, , Gaussian 09 Revision D.01, Gaussian Inc., Wallingford CT, 2009.

26. P. J. Stephens, F. J. Devlin, C. F. Chabalowski and M. J. Frisch, J. Phys. Chem., 1994, 98, 11623-11627.

27. V. A. Rassolov, M. A. Ratner, J. A. Pople, P. C. Redfern and L. A. Curtiss, J. Comput. Chem., 2001, 22, 976-984.

28. a) P. J. Hay and W. R. Wadt, J Chem Phys, 1985, 82, 299-310; b) P. J. Hay and W. R. Wadt, J Chem Phys, 1985, 82, 270-283; c) W. R. Wadt and P. J. Hay, J Chem Phys, 1985, 82, 284-298.

29. J. Kirkpatrick, Int. J. Quantum Chem., 2008, 108, 51-56.

30. a) K. Senthilkumar, F. C. Grozema, F. M. Bickelhaupt and L. D. A. Siebbeles, J. Chem. Phys., 2003, 119, 9809-9817; b) M. D. Newton, Chem. Rev., 1991, 91, 767-792. 